\title{
ANÁLISE DE MAPAS DE REPRESAS PUBLICADOS NA WEB USANDO IMAGENS ORBITAIS CCD/CBERS NO ESTADO DE MINAS GERAIS
}

\author{
ELIZABETH FERREIRA ${ }^{1}$, ANTONIO A. A. DANTAS ${ }^{2}$
}

RESUMO: Neste trabalho, bancos de dados públicos e gratuitos disponíveis na World Wide Web (WEB) foram utilizados para avaliar as áreas das superfícies dos espelhos d'água das represas de Furnas e do Funil, no Estado de Minas Gerais. O objetivo foi comparar as informações obtidas nos bancos da WEB com os valores das áreas calculadas a partir de imagens do sensor CCD a bordo dos satélites CBERS2 e CBERS2B. A área da represa de Furnas obtida a partir das imagens CCD/CBERS2B, ano 2008, foi de $1.138 \mathrm{~km}^{2}$, mas nos bancos de dados consultados esta área estava entre 1.182 e $1.503 \mathrm{~km}^{2}$. A represa do Funil, construída em 2003, com superfície de espelho d'água de $29,37 \mathrm{~km}^{2}$ e uma ilha com área de $1,93 \mathrm{~km}^{2}$ não aparecem nos bancos Atlas, Geominas, IGAM e IBGE. Os resultados mostraram algumas discrepâncias nos bancos de dados publicados na WEB, tais como diferenças em áreas e supressão ou extrapolação de limites do espelho d'água. Concluiu-se que, até o momento, os responsáveis por algumas publicações de bancos de dados no Estado de Minas Gerais não tiveram rigor suficiente com as atualizações. As imagens CCD/CBERS, que também são dados públicos disponíveis na WEB, mostraram ser produtos adequados para verificar, atualizar e melhorar as informações publicadas.

PALAVRAS-CHAVE: SIG, espelhos d'água, mapas digitais.

\section{ANALYSIS OF DAMS MAPS PUBLISHED ON THE WEB BY USING ORBITAL CCD/CBERS IMAGES IN MINAS GERAIS STATE, BRAZIL}

\begin{abstract}
In this work some public databases from the World Wide Web (WEB) were used to find the area of the Furnas and Funil Dams in Minas Gerais State. The purpose of this work was to compare the WEB public databases values and the real values obtained from the CCD camera images on board CBERS2 and CBERS2B satellites. The Furnas Dam area obtained from CCD/ CBERS2B images, in 2008, was $1.138 \mathrm{~km}^{2}$, but in the consulted databases this area ranged from 1.182 to $1.503 \mathrm{~km}^{2}$. The dam of the Funil, built in 2003, with a water surface of $29.37 \mathrm{~km}^{2}$ and an island with $1.93 \mathrm{~km}^{2}$ area, did not appear in Atlas, Geominas, IGAM and IBGE databases. The results revealed some problems in the WEB public databases like differences in the areas of dams and discrepancies like any non-existing border lines and sometimes extra areas. This suggested that some Brazilian WEB databases publishers have not been so far rigorous and the databases are outdated. The CCD/CBERS images that are WEB public databases showed to be suitable data for determining and updating the published information.
\end{abstract}

KEYWORDS: GIS, surface water, digital maps.

\section{INTRODUÇÃO}

O sensoriamento remoto e o geoprocessamento são áreas do conhecimento que se destinam à geração de produtos cartográficos e apresentam-se em diferentes estágios de desenvolvimento nos diversos países, conforme CHUVIECO (2002).

O desenvolvimento dessas tecnologias deve vir acompanhado pela produção de produtos cartográficos precisos e atuais. De acordo com GRANEMANN (2006), a cartografia básica de um país deve cumprir cinco pontos: estar atualizada; cobrir o país todo, sem exceção; cumprir com especificações técnicas que permitam representar as feições sem erros (que possam causar confusão

\footnotetext{
${ }^{1}$ Eng a Agrícola, Profa Dra., Departamento de Engenharia, UFLA, Lavras - MG, Fone: (0XX35)3829.1481, bethf@ufla.br

${ }^{2}$ Habilitado em Técnicas Agropecuárias, Prof. Dr., Departamento de Engenharia, UFLA, Lavras - MG.

Recebido pelo Conselho Editorial em: 4-5-2008

Aprovado pelo Conselho Editorial em: 13-10-2009

Eng. Agríc., Jaboticabal, v.30, n.1, p.110-120, jan./fev. 2010
} 
nos limites); existir em duas versões, ou seja, uma série cartográfica digital nacional e outra ortofotográfica, e garantir uma distribuição eficiente dos dados nas formas impressa e digital.

No Brasil, já são numerosos os usuários dos produtos cartográficos digitais. O mercado de produtos cartográficos na forma digital, facilitado pelo desenvolvimento dos Sistemas de Informações Geográficas (SIGs), Sistema de Posicionamento Global (GPS) e sensores digitais, tem alimentado, inclusive, uma política de acesso gratuito aos dados.

É consenso entre os usuários de SIG que a parte mais árdua e dispendiosa da análise de dados espaciais refere-se à construção e à alimentação do banco de dados, por isso há crescente preocupação em estabelecer soluções para facilitar o intercâmbio de informações. CAMBOIM (2006) destaca que, para cada tipo de informação (hidrografia, solos, arruamento, entre outros), existam uma ou mais instituições fazendo boa administração e atualização dos dados, sendo necessário, para tanto, o conhecimento específico e a jurisdição desses dados na esfera original. Portanto, mais eficiente do que duplicar os dados é conseguir pesquisar e compartilhar informações descentralizadas, diminuindo tarefas de retrabalho, como, por exemplo, a redigitalização de uma carta impressa em papel, já produzida em meio digital.

Outra questão a ser considerada é a atualização da informação. LOCH (2007) comenta sobre a deficiência e a desatualização do material cartográfico existente no Brasil, pois o mapeamento sistemático em escala 1:50.000 ou 1:100.0000 foi gerado em meados do século XX, o que evidentemente é inadequado para suportar avaliações em projetos de gestão territorial regional e local, considerando-se uma estrutura fundiária de minifúndios.

Entre as instituições e os órgãos responsáveis pela geração e pela publicação de mapas do Estado de Minas Gerais, destaca-se o Programa de Uso Integrado da Tecnologia de Geoprocessamento (Geominas), patrocinado pelo Governo de Minas Gerais. Um banco de dados do Geominas está disponível na página da Divisão de Processamento de Imagens do Instituto Espacial de Pesquisas Espaciais (DPI/INPE).

Na DPI/INPE, além de outros, também está disponível um banco denominado "atlas_acess", que é um atlas digital do Brasil com informações referentes aos Estados, municípios, cenas de satélite e hidrografia, além de outras.

Dentro da política de distribuição gratuita de dados, a Divisão de Geração de Imagens (DGI/INPE) disponibiliza imagens dos sensores dos satélites da série LANDSAT e dos Satélites Sino Brasileiro para Recursos Terrestres (CBERS2 e CBERS2B). O satélite CBERS2 foi lançado em outubro de 2003, e o CBERS2B, em setembro de 2007, levando a bordo câmeras que operam com diferentes resoluções espaciais, espectrais e temporais. A câmera de média resolução Charge Coupled Device (CCD) possui resolução espacial de $20 \mathrm{~m}$, gerando imagens em cinco faixas espectrais, com frequência de 26 dias. Os produtos da CCD são adequados para gerar cartas temáticas para fins de planejamento estadual e municipal, destacando-se a sua utilização em recursos hídricos para a delimitação de corpos de água e áreas alagadas.

A caracterização dos recursos hídricos de Minas Gerais é de responsabilidade do Instituto Mineiro de Gestão das Águas (IGAM). No seu banco de dados, pode-se encontrar a identificação de áreas específicas relacionadas a recursos hídricos.

O Instituto Brasileiro de Geografia e Estatística (IBGE) e a Divisão de Serviço de Cartografia do Exército (DSG) são os órgãos responsáveis pelo mapeamento sistemático do território brasileiro. O IBGE tem atribuições ligadas às geociências, destacando a coordenação dos sistemas estatísticos e cartográficos nacionais. Suas cartas topográficas em papel, elaboradas na década de 1970, em escala 1:50.000, são amplamente utilizadas para estudos referentes aos recursos hídricos.

Como são várias as instituições que disponibilizam mapas digitais de recursos hídricos na WEB, muitas vezes as informações não são coincidentes, deixando dúvidas quanto à confiabilidade. 
Mesmo com os sensores a bordo dos satélites da série CBERS produzindo imagens constantemente, também não há referências sobre a atualização dessas informações.

Nesse contexto, o objetivo deste trabalho foi comparar as áreas e os limites das superfícies dos espelhos d'água das represas de Furnas e do Funil (MG), obtidos a partir de imagens do sensor CCD a bordo dos satélites CBERS2 e CBERS2B com os dados de domínio público e gratuitos disponíveis na WEB, verificando a acessibilidade, a confiabilidade e a atualização dessas informações.

\section{MATERIAL E MÉTODOS}

Este trabalho foi dividido em três fases: na primeira, buscaram-se dados cartográficos digitais públicos e gratuitos disponíveis na WEB; na segunda, elaboraram-se os mapas das superfícies dos espelhos d'água das represas, utilizando-se imagens CCD/CBERS, e na terceira, os dados da WEB foram manipulados e comparados com os mapas obtidos a partir das imagens CCD/CBERS.

\section{Área de Estudo}

As represas de Furnas e do Funil estão localizadas em Minas Gerais entre as coordenadas geográficas $20^{\circ} 00^{\prime}$ e $21^{\circ} 45^{\prime}$ de latitude sul $46^{\circ} 30^{\prime}$ e $44^{\circ} 45^{\prime}$ de longitude oeste de Greenwich, conforme apresentado na Figura 1.
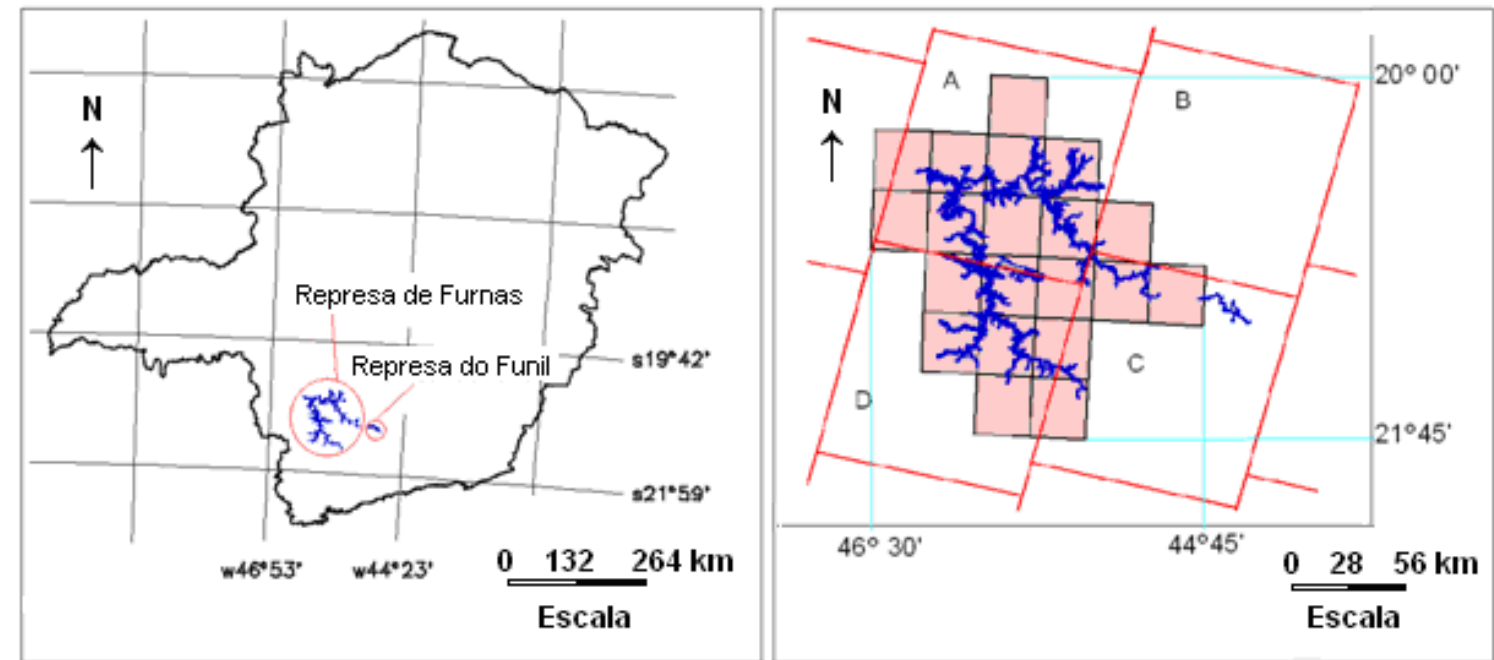

FIGURA 1. À esquerda, localização das represas em Minas Gerais. À direita as cores das grades representam as imagens dos satélites CBERS (vermelha) e as cartas do IBGE (preta). To the left localization of the dams in the state of Minas Gerais. To the right the colors of the grids represent the images of satellites CBERS (red) and the maps of the IBGE (black). WEB vector databases.

\section{Bancos de dados vetoriais na WEB}

$\mathrm{Na}$ Tabela 1, são apresentados as instituições, os dados obtidos, os anos em que foram gerados, os formatos dos dados e os endereços consultados na WEB.

A primeira busca de banco de dados vetoriais foi realizada na página do INPE. Desse endereço, foram copiados os bancos "geominas.exe" e "atlas_acess.exe", executáveis no software SPRING. O Sistema de Processamento de Informações Georreferenciadas (SPRING) é um SIG que integra a categoria de software livre, com distribuição gratuita via WEB. Tem funções de processamento de imagens, de modelagem numérica de terreno, de consulta a bancos de dados e de análise espacial, de acordo com CÂMARA et al. (1996). 
TABELA 1. Dados obtidos, ano, estrutura e endereço na WEB das instituições consultadas. Gotten data, year, structure and address on the WEB of the consulted institutions.

\begin{tabular}{cccc}
\hline Instituição & Dados Obtidos/Ano & $\begin{array}{c}\text { Estrutura/ } \\
\text { Formato }\end{array}$ & Endereço na WEB \\
\hline INPE & CBERS/2006-2008 & Imagem/tif & http://www.dgi.inpe.br/CDSR/ \\
NASA & Mosaico/2000 & Imagem/sid & https://zulu.ssc.nasa.gov/mrsisd \\
INPE & Geominas/1996 & Banco Spring & http:/www.dpi.inpe.br/spring \\
INPE & atlas_acess/1998 & Banco Spring & http:/www.dpi.inpe.br/spring \\
GEOMINAS & Represas/1996 & Vetorial/dxf & http://www.geominas.mg.gov.br/ \\
IGAM & Represas/2006 & Vetorial/dxf & http://www.igam.mg.gov.br/ \\
IBGE & Represas/1999 & Vetorial/dgn & http://www.ibge.gov.br/home/ \\
IBGE & Represas/1969 & Carta/papel & http://www.ibge.gov.br/home/ \\
IGA & Represas/1998 & Imagem/pdf & http://www.iga.br/mapas/cgi/iga_000.php \\
SIAM & Represas/1998 & Imagem/html & http://www.siam.mg.gov.br/siam/login.jsp \\
\hline
\end{tabular}

Neste trabalho, foram utilizados os dados do "Banco Geominas" da base de dados do INPE, projeto "Minas", ano de 1996, com projeção policônica e datum SAD-69, porque os mesmos já estavam no formato compatível com o software SPRING, minimizando esforços para construir um novo banco.

A partir desse passo, todos os arquivos de dados vetoriais, referentes à hidrografia e disponíveis na WEB, foram importados para o Banco Geominas, a fim de serem comparados com os resultados obtidos a partir das imagens.

Do "Banco Atlas", projeto "Brasil", projeção policônica, datum SAD-69, ano de 1998, também disponível no formato SPRING, foi importado o plano de informação (PI) de hidrografia.

Na página do IGAM, as informações são fornecidas em vários formatos vetoriais, sendo dada preferência ao formato "dxf”, por ser compatível com o SPRING. As informações estão disponíveis por categoria, sendo: distritos, hidrografia, localidades, sedes, série IBGE e unidades de planejamento. O tema hidrografia encontra-se subdividido em represas e cursos d'água, sendo importados do banco do IGAM para o projeto Geominas. Os dados do IGAM estão em projeção Geográfica e datum SAD-69, com escala original de 1:100.000 e 1:50.000, ano de 1998.

No endereço do IBGE, foram obtidas as cartas topográficas vetoriais, em escala 1:50.000, referentes ao mapeamento sistemático, com representações naturais e artificiais agrupadas por categoria de informação. Desse endereço, foram copiadas as cartas referentes à área de estudo, no total de 20 cartas (Figura 1). Após a descompactação dos dados, cada carta apresentou as seguintes categorias: hipsometria, hidrografia, localidades, sistema viário, limites, obras e edificações, pontos de referência e vegetação. A categoria hidrografia foi importada para o banco de dados Geominas. Essas cartas apresentam projeção Universal Transversa de Mercator (UTM) e datum Córrego Alegre, e foram digitalizadas pelo IBGE no ano de 1999. Ressalta-se que as mesmas se originaram de aerofotografias de 1964 que foram restituídas pelo IBGE em 1969.

Os outros portais consultados foram o do Instituto de Geociências Aplicadas (IGA) e o Sistema de Informações Ambientais (SIAM), no entanto, nesses endereços, não havia nenhuma permissão para download de dados vetoriais para serem utilizados em SIG, sendo possível apenas uma avaliação visual e qualitativa dos dados.

\section{Mapas gerados com a base nas imagens CBERS}

Para gerar os mapas das superfícies dos espelhos d'água a partir das imagens digitais dos satélites, foi necessária uma sequência de procedimentos que incluíram, entre outros, a aquisição, o georreferenciamento, a identificação ou interpretação das feições, a construção de mosaicos e as avaliações. 
Na Figura 1, pode ser vista a área de abrangência do conjunto de cenas que cobrem a área de estudo, cujas órbitas-ponto são: 154/123 (A), 153/123 (B), 153/124 (C) e 154/124 (D).

$\mathrm{Na}$ Tabela 2, estão especificados os satélites, o sensor, as bandas, as órbitas-ponto e as datas de obtenção das imagens. Essas imagens têm resolução espacial de 20 x $20 \mathrm{~m}$ e, nas datas em que foram obtidas, não havia presença de nuvens sobre as represas.

Para proceder ao georreferenciamento das imagens, deve-se utilizar uma base de dados confiável. Entre os serviços de imagens georreferenciadas, a National Aeronautics and Space Administration (NASA) disponibiliza os mosaicos de imagens do satélite ETM+/Landsat7 para praticamente toda a superfície terrestre. O mosaico S-23-20-2000 da NASA foi utilizado como base para georreferenciar as imagens CCD/CBERS.

TABELA 2. Especificações das imagens CCD/CBERS utilizadas no trabalho. Specifications of the CCD/CBERS images used in the work.

\begin{tabular}{cccc}
\hline Satélite/Sensor & Bandas & Órbita/Ponto & Data \\
\hline CBERS2/CCD & $1 ; 2 ; 3 \mathrm{e} 4$ & $154 / 123$ & $25-7-2006$ \\
CBERS2/CCD & $1 ; 2 ; 3 \mathrm{e} 4$ & $154 / 124$ & $25-7-2006$ \\
CBERS2/CCD & $1 ; 2 ; 3 \mathrm{e} 4$ & $153 / 124$ & $2-7-2006$ \\
CBERS2/CCD & $1 ; 2 ; 3 \mathrm{e} 4$ & $154 / 123$ & $28-6-2007$ \\
CBERS2/CCD & $1 ; 2 ; 3 \mathrm{e} 4$ & $154 / 124$ & $19-8-2007$ \\
CBERS2/CCD & $1 ; 2 ; 3 \mathrm{e} 4$ & $153 / 124$ & $5-6-2007$ \\
CBERS2B/CCD & $1 ; 2 ; 3 \mathrm{e} 4$ & $154 / 123$ & $14-6-2008$ \\
CBERS2B/CCD & $1 ; 2 ; 3 \mathrm{e} 4$ & $154 / 124$ & $14-6-2008$ \\
CBERS2B/CCD & $1 ; 2 ; 3 \mathrm{e} 4$ & $153 / 124$ & $13-7-2008$ \\
CBERS2B/CCD & $1 ; 2 ; 3 \mathrm{e} 4$ & $153 / 123$ & $13-7-2008$ \\
\hline
\end{tabular}

O georreferenciamento das imagens CCD/CBERS2, dos anos de 2006 e 2007, foi feito pela captura de pontos de controle no mosaico da NASA, pelo método imagem-imagem, com o uso do software SPRING. Para as imagens CCD/CBERS2B, do ano de 2008, foi utilizado o software ENVI (Environment for Visualizing Images), que possui rotina automática para georreferenciar imagens CCD/CBERS, utilizando como referência os mosaicos da NASA.

As imagens georreferenciadas de cada ano foram unidas por suas áreas comuns, construindo-se mosaicos. A análise dos mosaicos georreferenciados permitiu verificar se a área de estudo tinha sido totalmente coberta pelas imagens selecionadas.

Para delimitar as represas, inicialmente, foi feita uma classificação digital das imagens georreferenciadas pelo método supervisionado, adquirindo-se amostras de treinamento nas represas (água). Após o treinamento, aplicou-se o critério de máxima verossimilhança para separar a classe "água”, obtendo-se vetores, na forma de polígonos, que delimitavam os corpos d'água.

Em seguida, foi realizado um processo de edição em que os polígonos foram redigitalizados, ajustados e apagados, extraindo-se apenas os limites das represas de Furnas e do Funil, baseando-se na interpretação visual dessas represas nas imagens originais. As avaliações das represas e das ilhas foram, por fim, efetuadas.

\section{RESULTADOS E DISCUSSÃO}

Na Figura 2, podem ser vistas as represas de Furnas e do Funil em um mosaico de imagens CCD/CBERS2B, banda 4, ano de 2008, agora em projeção UTM, fuso $23 \mathrm{~K}$. 


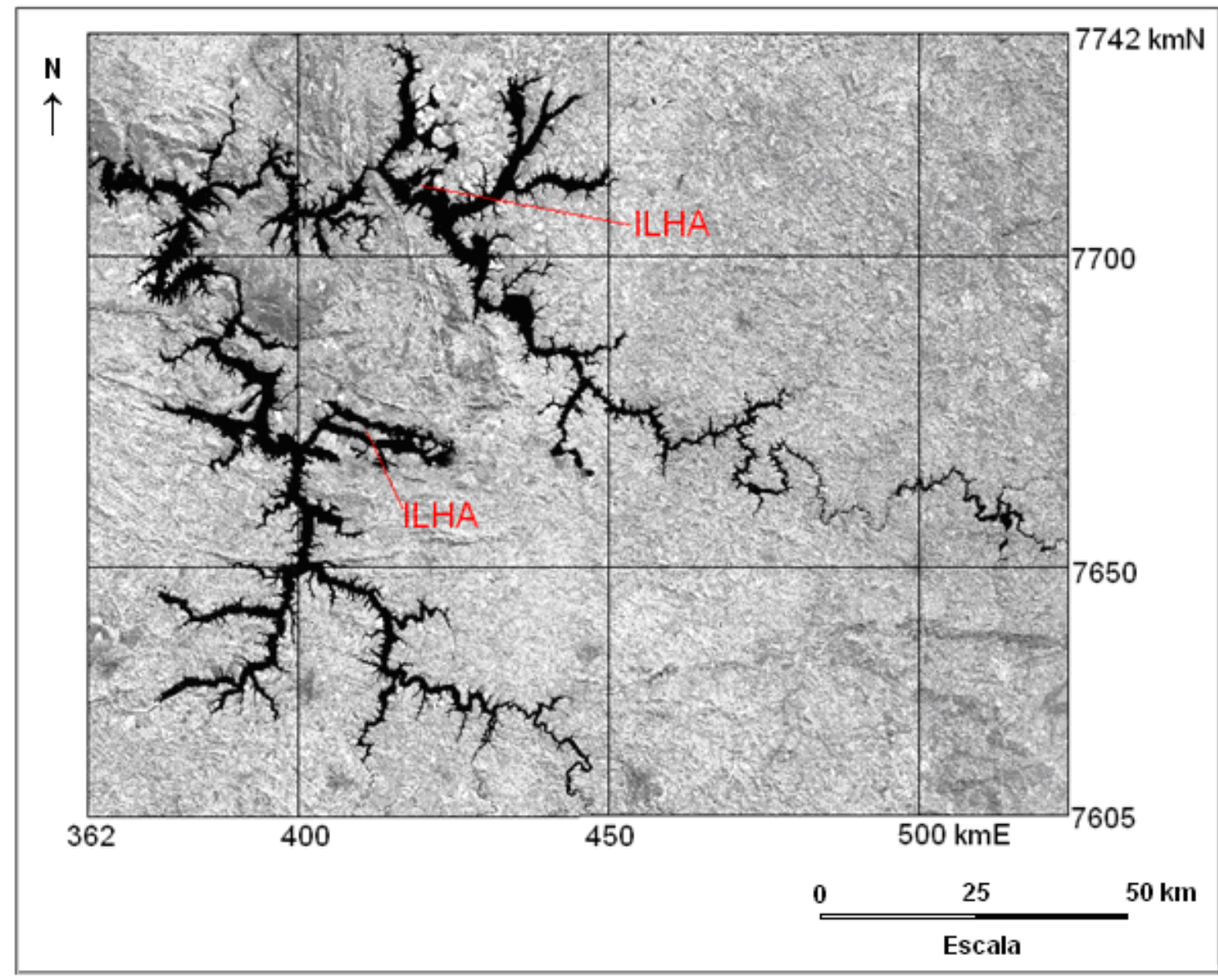

FIGURA 2. Mosaico de imagens CCD/CBERS2B, banda 4, ano de 2008. Mosaic of CCD/ CBERS2B images, band 4, year 2008.

\section{Represa de Furnas}

As áreas das superfícies dos espelhos d'água obtidas a partir das imagens CCD/CBERS2 e CCD/CBERS2B, para a Represa de Furnas, foram 1.120; 1.138 e $1.138 \mathrm{~km}^{2}$, para os anos de 2006, 2007 e 2008, respectivamente. A represa possui 12 ilhas, totalizando 19,30 km em área. A maior delas está situada nas coordenadas UTM $412 \mathrm{~km} \mathrm{E} \mathrm{e} 7.676 \mathrm{~km} \mathrm{~N}$, fuso $23 \mathrm{~K}$, com área de $13,63 \mathrm{~km}^{2}$. Outra ilha com área expressiva de $3,95 \mathrm{~km}^{2}$ está localizada nas coordenadas UTM $423 \mathrm{~km}$ E e $7.713 \mathrm{~km} \mathrm{~N}$. Essas duas ilhas estão indicadas na Figura 2.

No banco Atlas, após pequenos ajustes nas linhas que formavam o limite da represa, obteve-se área de $1.505 \mathrm{~km}^{2}$ para a represa de Furnas. Porém, o limite da represa encontra-se muito generalizado, sem qualquer detalhamento, concluindo-se que esse não deve ser utilizado como referência para cálculo de áreas e delimitação de represas.

A área da represa de Furnas obtida no projeto Geominas para os dados disponibilizados na WEB, no ano de 2006, foi de $1.433 \mathrm{~km}^{2}$. A mesma área disponibilizada no banco de dados do IGAM, no mesmo ano, totalizava $1.182 \mathrm{~km}^{2}$. Os limites das represas apresentados por esses projetos não foram totalmente coincidentes com o limite extraído da imagem CCD/CBERS, sendo que o Geominas apresentou maiores diferenças em termos de valor e de coincidência de área. Na Figura 3, podem ser vistos os limites de uma parte da represa de Furnas extraídos da imagem CCD/CBERS2B, do banco Geominas e do banco do IGAM. 


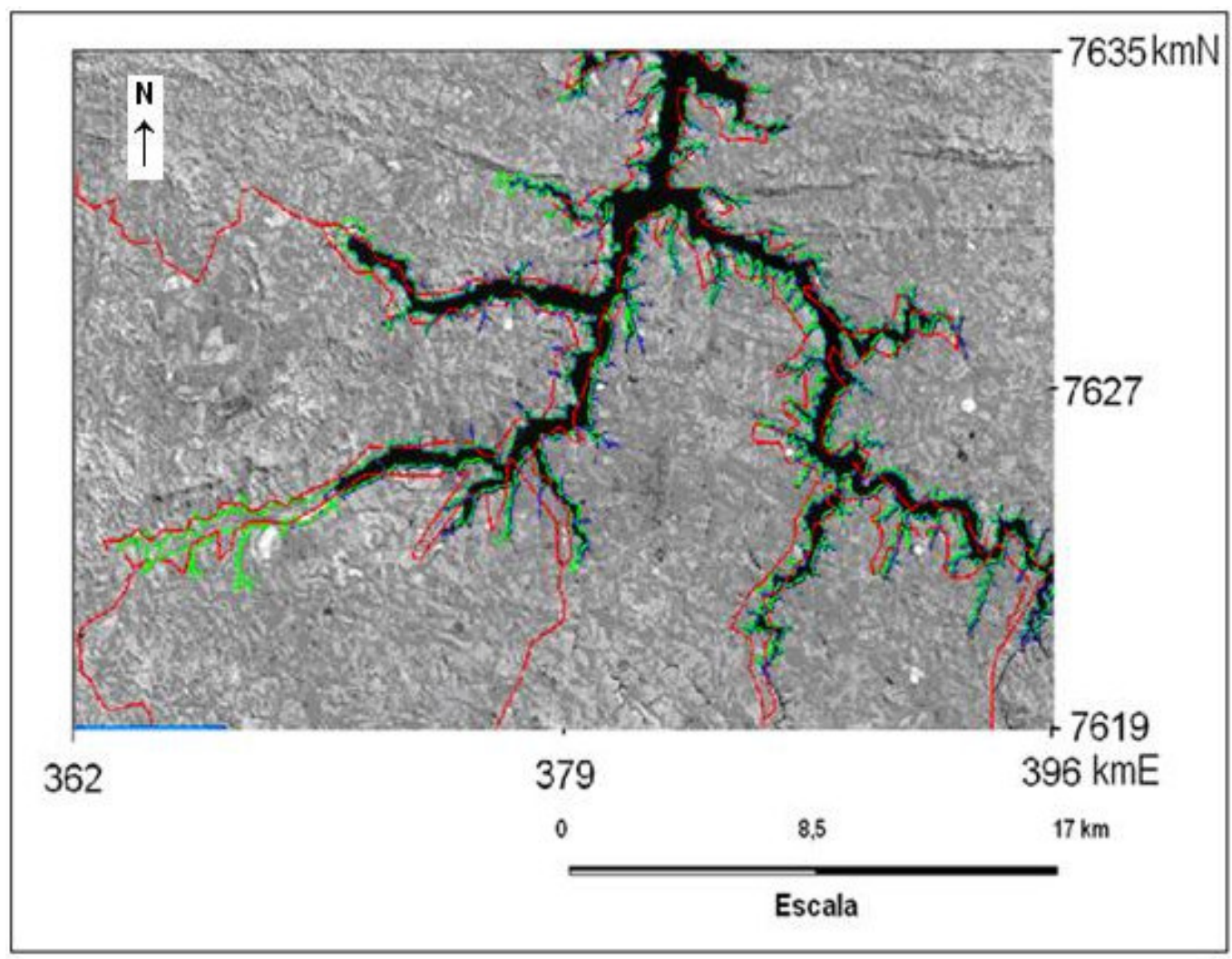

FIGURA 3. Limite de parte da Represa de Furnas extraído da imagem CCD/CBERS2B (cor preta e azul), do banco Geominas (linha vermelha) e do banco do IGAM (linha verde). Part limit of the Furnas Dam extracted from CCD/CBERS2B image (black and blue color), from Geominas database (red line) and from IGAM database (green line).

Analisando o limite extraído do banco Geominas, em todo o entorno, verificou-se que, tal como no banco Atlas, a linha do limite está muito generalizada, não devendo, portanto, ser utilizada para representar e muito menos para avaliar a área dessa represa.

De forma geral, verificou-se que o limite da represa de Furnas importado do banco do IGAM foi bastante coincidente com o mapa obtido a partir das imagens CCD/CBERS2. Porém, foram observadas regiões em que houve extrapolação de limites e regiões com supressão de áreas.

Na Figura 3, pode ser observada uma área em que o limite da represa foi além da superfície atual (imagens CCD/CBERS2) do espelho d'água. Essa área de represa inexistente corresponde a $16,27 \mathrm{~km}^{2}$. Para averiguar essa diferença, foram utilizados os dados digitais do IBGE que, dentre todos os bancos estudados, possui a maior escala (1:50.000). No entanto, nesse material, a represa está apresentada junto com as linhas de drenagem, conforme pode ser visto na Figura 4. Para determinar o limite e calcular a área da represa, seriam necessários muitos ajustes e redigitalizações, devido ao excesso de linhas e nós presentes na carta topográfica digitalizada (Figura 4). Essas redigitalizações poderiam modificar a estrutura original do banco do IBGE.

Diante desse fato, foi feita uma consulta ao material cartográfico impresso do IBGE, que corresponde às cartas denominadas Areado e Monte Belo, constatando-se que essa área específica está classificada como brejo ou pântano. Na imagem CCD/CBERS2, identifica-se facilmente que o espelho d'água termina e há início de uma área sujeita à inundação. Situações semelhantes ocorreram para as cartas de Capitólio e Nepomuceno. 


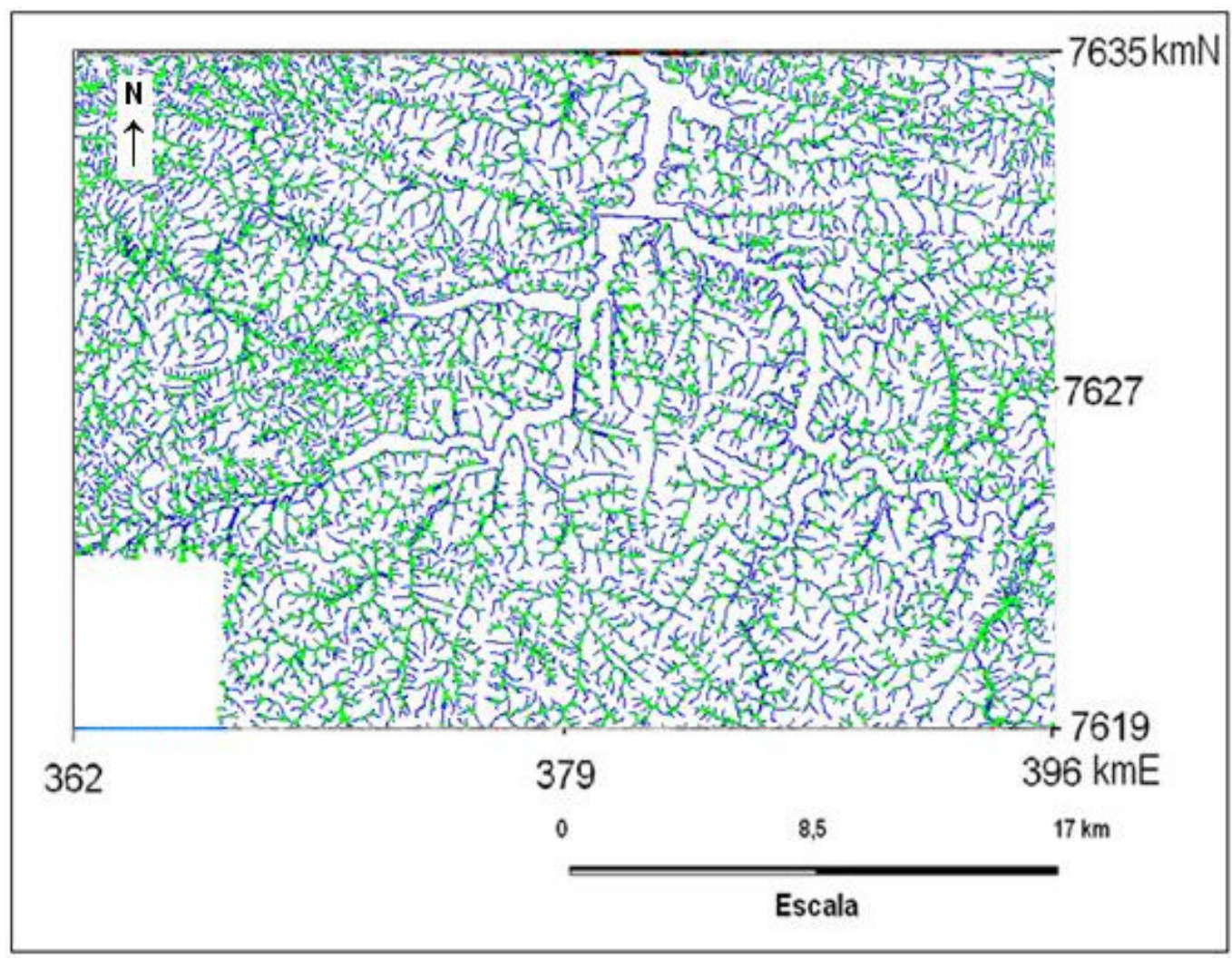

FIGURA 4. Carta topográfica digitalizada do IBGE com a rede de drenagem e a Represa de Furnas. IBGE digital topographic map with drainage lines and Furnas Dam.

O limite da represa apresentado no banco do IGAM coincide com o limite apresentado na carta do IBGE. Mas ambos diferem do mapa elaborado a partir da imagem CCD/CBERS2. Na carta impressa do IBGE, no Distrito de Pontevila, dentro do Município de Formiga, uma área significativa estava identificada como brejo. $\mathrm{Na}$ imagem CBERS atual, essa área aparece como água.

No banco do IGAM, as ilhas não estão mapeadas, e a ilha que possui a maior área aparece como se fosse um cabo. No entanto, no banco digital do IBGE, a maioria das ilhas está digitalizada e exatamente como as apresentadas no mapeamento gerado a partir das imagens CCD/CBERS2.

Pela análise desses bancos disponíveis na WEB, pode-se constatar que a coincidência ou discrepância entre os mapeamentos não é uma constante, mas há muita diferença entre os dados apresentados. FERREIRA et al. (2007) compararam dados sobre os limites e áreas dos municípios do entorno da Represa de Furnas em diferentes bancos de dados disponibilizados na WEB e verificaram que as informações não eram coincidentes e algumas vezes apresentaram-se erradas.

Outra questão a ser analisada refere-se à dificuldade de manipulação dos dados disponíveis, uma vez que, muitos desses, para serem utilizados, precisaram passar por diversas transformações. Essa troca de dados e informações é denominada interoperabilidade, sendo definida na ISO 19119, que trata de Serviços de Informação Geográfica, como a capacidade de comunicar, executar programas ou transferir dados entre várias unidades funcionais de modo que ao usuário seja requerido pouco ou nenhum conhecimento das características particulares de cada uma das unidades. Com certeza, após manipular e analisar os dados disponíveis na WEB, pode-se afirmar que é exigido do usuário bastante conhecimento nas áreas de geoprocessamento e processamento de imagens, faltando ainda a interoperabilidade.

Para os usuários que ainda não têm acesso ou habilidade para trabalhar com mapas digitais, as cartas impressas do IBGE continuam sendo uma boa fonte de consulta sobre a Represa de Furnas. 
Apesar de essas cartas terem sido confeccionadas na década de 1970, a superfície da represa, para a época estudada, não diferiu muito da apresentada neste material. Conforme GRANEMANN (2006), a cartografia básica de um país deve existir em duas versões, ou seja, uma série cartográfica digital e outra ortofotográfica, e garantir uma distribuição eficiente dos dados nas formas impressa e digital, além de estar atualizada.

Com relação aos bancos de dados do IGA e do SIAM, procedeu-se apenas uma avaliação qualitativa dos dados visualizados em tela, em 19-4-2008, verificando-se, também, que o contorno da represa de Furnas, em ambos os bancos, comparado com o obtido neste trabalho (CCD/CBERS2), não foi coincidente, estando desatualizado em algumas regiões.

\section{Represa do Funil}

A escolha da Represa do Funil atribuiu-se ao fato de essa ter sido construída no ano de 2003 e não constar em mapas antigos, sendo um referencial para avaliar a atualização da informação disponível na WEB. A represa do Funil possui superfície de espelho d'água de $29,37 \mathrm{~km}^{2}$ e uma ilha com área de $1,93 \mathrm{~km}^{2}$ (Figura 5).

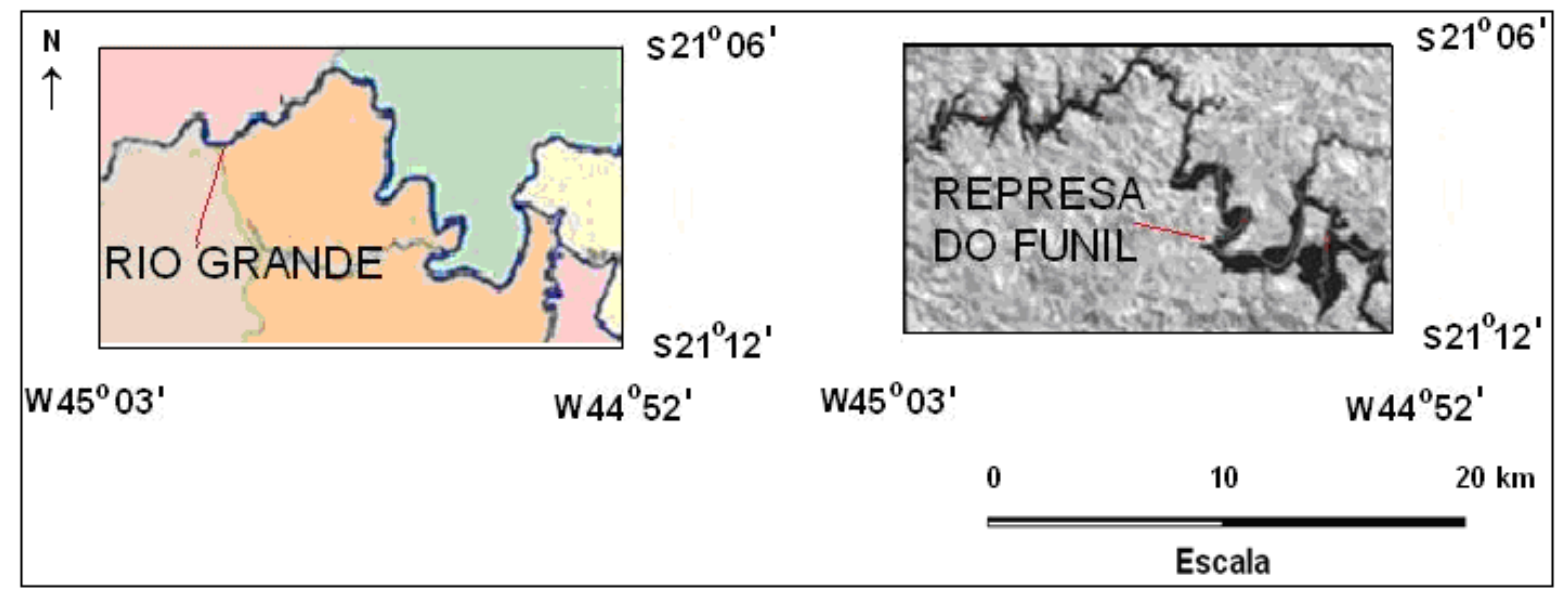

FIGURA 5. Mapa do IGA sem a representação da represa, apenas do Rio Grande, e imagem CCD/CBERS2, banda $4 \mathrm{com}$ a Represa do Funil. IGA map without the representation of the dam, just Rio Grande, and Funil Dam in an image CCD/CBERS2, band 4.

Nos bancos Atlas, Geominas, IGAM e IBGE não constam dessa represa. Mesmo bem visível na imagem orbital, não apareceu no mapeamento do IGA, confirmando que a informação está desatualizada. Na Figura 5, está o mapa do IGA (19-4-2008) apresentando o Rio Grande ainda sem a Represa do Funil. A mesma está realçada na cor preta em imagem de satélite CCD/CBERS2 de 2-7-2006. Ressalta-se que a represa foi construída em 2003.

No portal do SIAM, estão disponíveis os resultados do estudo do Zoneamento Ecológico Econômico (ZEE). Nesse portal, são apresentados dentro da categoria hidrografia os planos de informação represa, cursos d'água, bacias, sub-bacias, microbacias e bacias em conflitos. Apesar de esses estudos serem recentes (2007), os autores também não representaram a Represa do Funil. Semelhante aos mapas apresentados nos outros projetos consultados, o tema ainda está desatualizado nesse banco de dados.

Várias iniciativas têm sido tomadas, pelo poder público, para suprir a necessidade de atualização dos dados para fins de planejamento. A Agência Nacional das Águas (ANA) está realizando mapeamento dos espelhos d'água do Brasil utilizando imagens CCD/CBERS2, e para o Estado do Ceará conseguiu-se mapear espelhos com área de $0,2 \mathrm{~km}^{2}$, ou seja, 20 hectares. 


\section{CONCLUSÕES}

Em vista das análises e comparações realizadas sobre alguns dados públicos e gratuitos na WEB, no período de 2006 a 2008, verificou-se que, embora se tenha uma quantidade considerável de dados disponibilizados por diversas instituições, as informações estão desatualizadas, não são coincidentes e algumas vezes estão erradas.

As imagens do sensor CCD a bordo do satélite CBERS2 podem ser utilizadas para a atualização de superfícies de espelho d'água, enfatizando e validando a importância do projeto sino-brasileiro e sua política de distribuição gratuita dos dados.

\section{AGRADECIMENTOS}

À Fundação de Amparo à Pesquisa do Estado de Minas Gerais (FAPEMIG), processo SHAAPQ-2925-3-12/07.

\section{REFERÊNCIAS}

CÂMARA, G.; SOUZA, R.C.M.; FREITAS, U.M.; GARRIDO, J. Spring: Integrating remote sensing and GIS by object-oriented data modelling. Computers \& Graphics, v.20, n.3, p.395-403, 1996.

CAMBOIM, S.P. A questão da interoperabilidade. Infogeo, Curitiba, n.43, p.26-27, 2006.

CBERS CCD. São José dos Campos: Instituto Nacional de Pesquisas Espaciais, 2006-2008. Imagem de satélite. Canais 1, 2, 3 e 4.

CHUVIECO, E. Teledection ambiental: la observación de la tierra desde el espacio. Barcelona: Ariel, 2002. $586 \mathrm{p}$.

DPI/INPE. INSTITUTO DE NACIONAL DE PESQUISAS ESPACIAIS. Disponível em: $<$ http://www.dpi.inpe.br/spring/portugues/index.html> Acesso em: jul. 2006.

FERREIRA, E.; DANTAS, A.A.A.; CARVALHO, M.A.de. Avaliação da área e do entorno da Represa de Furnas - MG, por meio de SIG utilizando dados públicos: facilidades, dificuldades e problemas. In: SIMPÓSIO BRASILEIRO DE SENSORIAMENTO REMOTO, 13., 2007, Florianópolis. Anais... São José dos Campos: Instituto Nacional de Pesquisas Espaciais, 2007. 1 DVD.

GRANEMANN, E. A revolução do conhecimento. Infogeo, Curitiba, n.40, p.32-33, 2006.

IBGE. INSTITUTO BRASILEIRO DE GEOGRAFIA E ESTATÍSTICA. Disponível em: $<$ http://www.ibge.gov.br/home/geociencias/cartografia/default.shtm> Acesso em: jun. 2006, mar. 2008.

IGA. INSTITUTO DE GEOCIÊNCIAS APLICADAS. Disponível em: http://www.iga.br/mapas/cgi/IGA_Cartografia/IGA_Cartografia.php. Acesso em: 19 abr. 2008

IGAM. INSTITUTO MINEIRO DE GESTÃO DAS ÁGUAS. Disponível em: http://www.igam.mg.gov.br/. Acesso em: ago. 2006, mar. 2008.

INTERNATIONAL ORGANIZATION FOR STANDARTIZATION. Disponível em: <http://www.iso.org/iso/en/ISOOnline.frontpage>. Acesso em: jun. 2006.

LOCH, C. A realidade do cadastro técnico multifinalitário no Brasil. In: SIMPÓSIO BRASILEIRO DE SENSORIAMENTO REMOTO, 13., 2007, Florianópolis. Anais... São José dos Campos: Instituto Nacional de Pesquisas Espaciais, 2007. 1 DVD.

NASA. Earth Science Applications Directore-MRSid Image Server. Disponível em: $<$ https://zulu.ssc.nasa.gov.mrsid>. Acesso em: maio 2006. 
PARMA, G.C. Mapas cadastrais na internet: servidores de mapas. In: SIMPÓSIO BRASILEIRO DE SENSORIAMENTO REMOTO, 13., 2007, Florianópolis. Anais... São José dos Campos: Instituto Nacional de Pesquisas Espaciais, 2007. 1 DVD.

SIAM. SISTEMA DE INFORMAÇÃO AMBIENTAL. Disponível em:

<http://www2.siam.mg.gov.br/webgis/zee/viewer.htm>. Acesso em: 18 abr. 2008 